\title{
Gaugino Condensation and Nonperturbative Superpotentials in Flux Compactifications
}

\author{
Lars Görlich $^{a}$, Shamit Kachru ${ }^{b}$, Prasanta K. Tripathy ${ }^{a}$ and Sandip P. Trivedi ${ }^{a 1}$ \\ a Tata Institute for Fundamental Research \\ Homi Bhabha Road, Mumbai 400 005, INDIA \\ ${ }^{b}$ Department of Physics and SLAC \\ Stanford University \\ Stanford, CA 94305/94309, USA
}

There are two known sources of nonperturbative superpotentials for Kähler moduli in type IIB orientifolds, or F-theory compactifications on Calabi-Yau fourfolds, with flux: Euclidean brane instantons and low-energy dynamics in D7 brane gauge theories. The first class of effects, Euclidean D3 branes which lift in M-theory to M5 branes wrapping divisors of arithmetic genus 1 in the fourfold, is relatively well understood. The second class has been less explored. In this paper, we consider the explicit example of F-theory on $K 3 \times K 3$ with flux. The fluxes lift the D7 brane matter fields, and stabilize stacks of D7 branes at loci of enhanced gauge symmetry. The resulting theories exhibit gaugino condensation, and generate a nonperturbative superpotential for Kähler moduli. We describe how the relevant geometries in general contain cycles of arithmetic genus $\chi \geq 1$ (and how $\chi>1$ divisors can contribute to the superpotential, in the presence of flux). This second class of effects is likely to be important in finding even larger classes of models where the KKLT mechanism of moduli stabilization can be realized. We also address various claims about the situation for IIB models with a single Kähler modulus.

July 2004

\footnotetext{
1 emails: goerlich, prasanta, sandip @theory.tifr.res.in, skachru@stanford.edu
} 


\section{Introduction and Review}

For many years, it has been clear that string theory offers a plethora of choices for compactification to $4 \mathrm{~d}$ with $\mathcal{N} \geq 1$ supersymmetry. With $\mathcal{N}=1$ supersymmetry, quantum effects can play an important role in breaking supersymmetry and/or changing the vacuum structure. It has long been hoped that such effects, perhaps in conjunction with early universe dynamics, would yield one or a few models as the preferred string compactifications - i.e., that there would be a simple vacuum selection principle. There is little evidence for such a picture (though of course our understanding of early universe cosmology in string theory is very limited). Instead, recent attempts to understand moduli stabilization have yielded mounting evidence that after including perturbative and nonperturbative contributions to the moduli potential, string theory manifests a tremendous landscape of vacua, including 4d (metastable) de Sitter and anti-de Sitter geometries with a wide range of different cosmological constants [1-5]. For nice reviews of this subject, see [6]. While a similar picture should emerge in each of the corners of the M-theory parameter space, we will concern ourselves here with the type IIB theory, where the story is best developed. Because our goal in this paper is to clarify and extend some recent developments in this area, we will begin with a short review and status report on the subject.

If one wishes to obtain theories with $4 \mathrm{~d} \mathcal{N} \leq 1$ supersymmetry in the IIB setting, one large class of constructions was developed in [7] and references therein. These models are Calabi-Yau orientifolds with D3 and/or D7 branes, and also admit a description as Ftheory compactified on a Calabi-Yau fourfold. In a given such construction corresponding to compactification on the threefold $M$ (with related fourfold $X_{4}$ ), one finds a tadpole condition which requires that the total D3 brane charge on $M$ add up to zero [8]

$$
N_{D 3}+\frac{1}{(2 \pi)^{4}\left(\alpha^{\prime}\right)^{2}} \int_{M} H \wedge F=\frac{\chi\left(X_{4}\right)}{24} .
$$

Here $H$ and $F$ are the NS and RR three-form field strengths of the IIB theory, and $N_{D 3}$ denotes the number of D3 branes one has chosen to insert transverse to $M$. So we see that in generic backgrounds, one will turn on RR and NS fluxes as part of tadpole cancellation.

The resulting class of $4 \mathrm{~d} \mathcal{N}=1$ supergravities was described in [7]. The fluxes generate a superpotential for the complex structure moduli and the dilaton $\tau$. Defining $G_{3}=F-\tau H$, this superpotential is of the form [9]

$$
W_{f l u x}=\int_{M} G_{3} \wedge \Omega
$$


where $\Omega$ is the holomorphic three-form on $M$. For typical choices of the flux, the dilaton and complex structure moduli have isolated minima.

On the other hand, the Kähler moduli $\rho^{i}$ of $M$ do not appear in $W$, and participate in a no-scale cancellation at leading orders in the $\alpha^{\prime}$ and $g_{s}$ expansion [7]. One generally expects that quantum corrections will generate a potential for the $\rho$ fields. If this potential arises only from corrections to the Kähler potential, any nontrivial vacua will typically occur for string scale compactification manifolds, and will be difficult to study.

As emphasized in [3], however, there are at least two classes of effects that lead one to expect $\rho$-dependent corrections to the superpotential in many models:

1) If $M$ contains a 4 -cycle $\Sigma$ with the right topological properties, Witten argued that Euclidean D3 branes will generate a nonperturbative superpotential for the Kähler modes controlling the size of $\Sigma[10]$. These cycles lift, in the M-theory fourfold geometry, to "vertical" divisors of arithmetic genus 1 (where "vertical" roughly denotes that they wrap the fiber directions which shrink in the F-theory limit).

2) These models typically contain D7 branes. While as discussed in [11] the D7s often have numerous matter fields in the most naive Kaluza-Klein analysis of their $4 \mathrm{~d} \mathcal{N}=1$ supersymmetric worldvolume gauge theory, the three-form flux can give masses to many or all of these matter fields. If the fluxes do this while stabilizing the D7s at a coincident locus, gaugino condensation will ensue. For $N$ D7s wrapping a 4-cycle $\Sigma$, the gauge coupling will satisfy $\frac{1}{g^{2}} \sim \operatorname{Vol}(\Sigma)$, and therefore the gauge theoretic superpotential will generate a nonperturbative potential for Kähler moduli.

In the approximation that one keeps the leading contribution to this superpotential, one gets a schematic formula of the form

$$
W=W_{f l u x}+e^{-a \rho}
$$

If $W_{\text {flux }}$ evaluated in the vacuum of the complex structure and dilaton moduli is small, i.e. $W_{\text {flux }}=W_{0}<<1$, then one finds a resulting vacuum for $\rho$ at moderately large volume. For instance in [3], a toy example was described that, with $W_{0}$ of $10^{-4}$ and $a$ of $\frac{1}{10}$, achieved a radius of just above 3 in string units (which translates to $\rho \sim 100$ ). These moderately large radii can justify the neglect of more highly damped exponentials in the formula (1.3), yielding self-consistent solutions to the equations of the effective field theory. The existence of solutions with $W_{0}<<1$ was justified in [3] by explaining that given the number of flux vacua and naive estimates for how $W_{0}$ might vary in different solutions, very small values should arise in a small fraction of the solutions. 
The vacua just described are, in the simplest cases, supersymmetric AdS vacua. It was further argued in [3] that by e.g. using warped solutions of the sort described in [7] (which incorporate a Klebanov-Strassler throat [12]), and including anti-D3 branes (whose dynamics in such throats was studied in [13]), one should be able to obtain de Sitter solutions to string theory. Instead of including anti-D3 branes, one could also imagine using anti-self dual field strengths in D7 branes [14]. Alternatively, one can simply start in a vacuum of the no-scale potential which is at positive $V$, and play off the tadpole for Kähler moduli against the nonperturbative corrections [15]. Another possibility was described in [16]. The end result, as argued in [3] and later references, is that the IIB superstring theory seems to admit a rich landscape of vacua with many de Sitter and antide Sitter critical points, exhibiting different values of the cosmological constant. While only a small fraction of the vacua will arise from small $W_{0}$ in the language above, and will therefore arise at moderately large radius, this small fraction was argued to yield a large absolute number of vacua.

Two recent lines of development have added considerable support to this picture. In the first, Ashok and Douglas and later authors [17-19] have studied the statistics of the flux vacua which arise in the no-scale approximation. The most basic result concerns the number of vacua, and finds (as one would expect from simple generalizations of [1]) that

$$
N_{v a c} \sim \frac{1}{b^{3}(M) !}\left(\frac{\chi\left(X_{4}\right)}{24}\right)^{b^{3}(M)}
$$

Typical numbers yield $\frac{\chi}{24}$ of order 1000 and $b^{3}(M)$ of order 100, and in fact $N_{v a c}$ can be in excess of $10^{300}$ in simple examples. While these estimates neglect many possible further effects that could remove vacua, the basic picture seems robust against effects that have been neglected to date. Estimates of the attainable values of $W_{0}$ (or really $e^{K}|W|^{2}$ ) at the level of the flux superpotential, fully support the assertion made in [3] that extremely small values will be attainable. In fact, values much smaller than the $10^{-4}$ quoted in KKLT should arise - the fraction of vacua with $e^{K}|W|^{2} \leq \epsilon$ seems to fall off only as the first power of $\epsilon[18,20]$. The information available from the statistical studies is much more detailed than we have described here (predicting for instance clustering of vacua around the conifold [18,19] and other interesting dynamics), and it is heartening that the extremely complicated flux potentials admit such simple characterizations of the statistical properties of the resulting vacua. 
However, one could still worry that although each of the ingredients in the KKLT construction and its relatives is quite reasonable, it might not be possible to assemble all of the ingredients simultaneously to make a working model. While this potential problem seems very unlikely from the viewpoint of low-energy effective field theory, the issue has now been settled directly from the string theory perspective. In a beautiful recent paper, Denef, Douglas and Florea (henceforth DDF) explicitly provided many examples of CalabiYau orientifolds where just Euclidean D3 instantons (effects of type 1) above) are present in sufficient numbers to stabilize all Kähler moduli [11]. Their constructions build on earlier important work of Grassi [21]. These models admit sufficiently many flux vacua that the full KKLT construction can be carried through, as long as the existing statistical studies are not grossly misleading. The fraction of models where just effects of type 1) suffice to carry out the KKLT program is not particularly small - in the simplest class DDF studied (fourfolds with Fano threefold base), 29 of 92 Calabi-Yau spaces could be stabilized this way. Each such manifold leads to a rich landscape of vacua. A subsequent work [22] also pointed to the existence of manifolds which cannot be stabilized by such effects alone, although this work did not address the large class, described in [11], which can be stabilized.

Therefore, current evidence strongly supports the existence of a IIB landscape as envisioned in [3]. Among many issues which remain to be clarified is the question of the extent to which effects of type 2) above, low-energy field theory effects on D7 branes, can aid in the stabilization of Kähler moduli. In this work, we provide some explicit compact examples where flux potentials lift D7 translation modes, leaving a pure nonabelian $\mathcal{N}=1$ gauge theory on stacks of D7s. These will manifest gaugino condensation, although they do not fall into the class of models studied in geometric engineering in [23]- the latter work did not (and did not need to) account for D7 interactions with three-form fluxes.

Before proceeding with the technical analysis, we give a simple physical argument which explains why one should expect the phenomena we find to be rather generic. The gauge theories which arise on D7 brane worldvolumes are, in most simple examples, nonchiral gauge theories. Even if a naive analysis at the compactification scale indicates the presence of matter in the theory (the relevant KK scale analysis is described in [23] and other references), any further interactions at a lower scale can therefore give the matter a mass, leaving behind a pure gauge theory. In these systems, there is a clear source of such further perturbations - the presence of $G_{3}$ flux. Physically, it is then not surprising that one will often find pure gauge theories after including the effects of flux. We provide 
explicit examples on the fourfold $K 3 \times K 3$ (the orientifold $K 3 \times T^{2} / Z_{2}$ in IIB language) in the following; this example was chosen because it is one of the simplest models that includes D7 branes, and was analyzed in great detail in [24] (see also [25]). For the physical reason we explained above and the mathematical reasons we explain below, we also suspect this phenomenon occurs frequently in more complicated models, and will greatly enrich the class of models described so far [11] where the KKLT construction can be realized.

The organization of this note is as follows. In $\S 2$, we review the conditions for supersymmetry in M-theory and F-theory compactification on a Calabi-Yau fourfold. In $\S 3$, we describe what these conditions imply for $K 3 \times K 3$ models in more detail. In $\S 4$, we provide some examples of solutions to these conditions which yield $4 \mathrm{~d}$ models with the D7 branes locked on loci of enhanced non-abelian gauge symmetry. In $\S 5$, we explore an orientifold example in detail and calculate the resulting non-perturbative superpotential. In $\S 6$, we discuss the kinds of divisors which arise in these singularities. In particular, there are no divisors of arithmetic genus 1 in the $K 3 \times K 3$ examples, although the relevant divisors $D_{i}$ do satisfy $\chi\left(D_{i}, \mathcal{O}\left(D_{i}\right)\right)>1$ (and in more general examples, would have $\chi \geq 1$ ). We also explain how these observations, when correctly generalized to other Calabi-Yau fourfolds, could relax some of the conditions stated in $[22,11]$. In particular, we briefly discuss the special case where the IIB theory has a single Kähler modulus, and argue that $\chi \geq 1$ divisors of the relevant type can arise there. We close in $\S 7$ with a discussion of future directions. Some relevant details about the geometry of elliptic $K 3$ s and the $E_{8}$ lattice are relegated to appendices, as is a discussion of how one can microscopically understand the relaxation of the arithmetic genus 1 condition [10] in the presence of flux.

The $D 7 / D 3$ moduli in the $K 3 \times T^{2} / Z_{2}$ orientifold limit were studied earlier in [26] using the techniques of gauged supergravity. In particular it was shown that $D 7$-brane moduli acquire a mass, in agreement with our discussion here. Many of the solutions found below were also known to Greg Moore, from considerations similar to those he described in [25]. A complementary approach to deriving the flux-induced potentials for D7-brane moduli is described in the papers $[27,28]$, and we thank those authors for informing us of their results prior to publication .

\section{General Conditions For Supersymmetry}

We are interested in F-theory compactifications on $K 3 \times K 3$ with flux. In particular we are interested in asking whether the flux can stabilise all the complex structure moduli 
(including the 7-brane moduli) at points of enhanced gauge symmetry. In the discussion below we take the second $K 3$ to be elliptically fibered and refer to it as $K 3_{2}$, the other is denoted as $K 3_{1}$ and need not admit an elliptic fibration.

We will explore this issue by starting first in $M$ theory on $K 3_{1} \times K 3_{2}$ in the presence of $G_{4}$ flux. This is dual to F-theory on $K 3 \times K 3 \times S^{1}$. We will be mainly interested in Lorentz invariant $3+1$ dimensional solutions in F-theory. These are obtained in the standard fashion by taking the size of the fiber torus in $K 3_{2}$ to zero.

For $\mathcal{N}=1$ supersymmetry (four supercharges) $G_{4}$ must be of type $(2,2)$. In addition $G_{4}$ must be primitive. For Lorentz invariant $3+1$ dimensional solutions $G_{4}$ must have one leg along the base $P^{1}$ and another leg along the fiber of $K 3_{2}$. Primitivity then reduces to the condition

$$
G_{4} \wedge J_{1}=0
$$

where $J_{1}$ is the Kähler form of $K 3_{1}$.

An elliptically fibered $K 3$ can be described by an equation of Weierstraß form:

$$
y^{2}=x^{3}+f_{8}(z) x+g_{12}(z),
$$

where $f_{8}(z)$ and $g_{12}(z)$ are polynomials of degree 8 and 12 respectively. This gives rise to 18 (complex) moduli which describe the complex structure of an elliptically fibered $K 3$ surface. It is well known that the singularities which can occur in this equation are of AD-E type. At a singularity the symmetry is enhanced to the corresponding A-D-E gauge group.

We will show in specific examples that for appropriate fluxes all the conditions of $\mathcal{N}=1$ supersymmetry are met and all the complex structure moduli are stabilised such that the elliptically fibered $K 3$ is at a singularity resulting in an enhanced gauge symmetry.

For this purpose it is worth discussing the above conditions in some more detail. Some useful reference for the discussion below are [29], [7], [30], and [24].

\section{The Conditions in More Detail}

$H^{2}(K 3, R)$ is a 22 dimensional vector space. An inner product can be defined on this space, given by $\left(v_{1}, v_{2}\right) \equiv \int_{K 3} v_{1} \wedge v_{2}$. This has signature $(3,19) . H^{2}(K 3, Z)$ can be thought of as a lattice, $\Gamma^{3,19}$, embedded in this vector space. In a suitable basis the inner product for the basis elements of this lattice takes the form of the matrix

$$
U \oplus U \oplus U \oplus\left(-E_{8}\right) \oplus\left(-E_{8}\right)
$$


where $U$ is the $2 \times 2$ matrix

$$
U=\left(\begin{array}{ll}
0 & 1 \\
1 & 0
\end{array}\right)
$$

and $E_{8}$ is the Cartan matrix for the $E_{8}$ lattice.

The holomorphic two-form $\Omega$ on $K 3$ is given by a spacelike oriented two-plane in $H^{2}(K 3, R)$. The moduli space of complex structures then corresponds to the space of all such distinct two-planes. Up to discrete identifications this is

$$
G=O(3,19) / O(2) \times O(1,19)
$$

which is 40 dimensional. In the discussion below we will refer to both the holomorphic two-form and the associated space-like two plane as $\Omega$. Clearly, all two-forms in $H^{(1,1)}$ must be orthogonal to $\Omega$.

For the $K 3$ surface to be elliptically fibered, a sublattice $U \subset \Gamma^{(3,19)}$ must be orthogonal to the two-plane ${ }^{2} \Omega$. The moduli space of complex structures for elliptically fibered $K 3 \mathrm{~s}$ is then given (again up to discrete identifications) by $O(2,18) / O(2) \times O(18)$. This is 18 (complex) dimensional, the counting agrees with the moduli in the Weierstraß form, (2.2). The Picard lattice of $K 3$ is defined as the lattice of integral two-forms which are of $(1,1)$ type. That is

$$
\operatorname{Pic}(K 3)=H^{2}(K 3, Z) \cap H^{(1,1)}(K 3) .
$$

So we see that for the $K 3$ to be elliptically fibered $U \subset P i c(K 3)$.

How this requirement for an elliptic fibration comes about will be discussed further in the Appendix. For now we simply note that the two-forms dual to the base $P^{1}$ and the fibre torus both lie in $U$. We saw above that $G_{4}$ must have one leg along the base and one along the fibre of the elliptically fibered $K 3$. This means that $G_{4}$ cannot contain any element in $U$ and so must be orthogonal to $U$. That is for any $u \in U, G_{4} \cdot u=0$.

We can now restate the conditions for supersymmetry as follows. $G_{4}$ must be chosen to have two legs along $K 3_{1}$ and two legs along $K 3_{2}$. A complex structure on $K 3_{1}$ and $K 3_{2}$ must exist such that $G_{4}$ is of type $(2,2)$. The resulting Picard lattice for elliptically fibered $K 3_{2}$ must contain the sublattice $U . G_{4}$ must be orthogonal to $U$. And finally, the Kähler form of $K 3_{1}$ must satisfy the primitivity condition, (2.1), which can be restated in terms of the inner product defined above as $J_{1} \cdot G_{4}=0$.

\footnotetext{
2 More accurately the sublattice has two basis elements whose inner product takes the form $U$, (3.1). In an abuse of notation we will refer to the sublattice itself as $U$ below.
} 
Let us now discuss the singularities in the Weierstraß form, (2.2), in some more detail. A root of $\Gamma^{3,19}$ is defined to be a vector $\alpha \in \Gamma^{3,19}$ with $\alpha \cdot \alpha=-2$. A singularity in the Weierstraß form arises if there is a root of $\Gamma^{3,19}$ which lies in Pic(K3) and which is orthogonal to $U$. That is, if there is a root orthogonal to both $U$ and $\Omega{ }^{3}$ The orthogonal roots form the root lattice of an A-D-E algebra. The singularity is of the corresponding A-D-E type.

Before proceeding, let us make the following two comments. First, it is worth briefly recapitulating why one expects all complex structure moduli to be generically stabilised in an $\mathcal{N}=1$ susy solution. The requirement that $G_{4}$ is of type $(2,2)$ means that the $(4,0),(0,4)$ and $(1,3),(3,1)$ components in $G_{4}$ must vanish. This imposes one more condition than the number of complex structure moduli. For a choice of flux where a susy solution does exist this implies that all the complex structure moduli should generically be lifted.

Second, the four-form flux, $G_{4}$, gives rise to three form flux in the IIB description. Let the holomorphic and anti-holomorphic differentials along the elliptically fibered torus of $K 3_{2}$ be $d z$ and $d \bar{z}$, and $\phi$ be the modular parameter (the axion-dilaton in the IIB theory). Then $G_{4}$ can be expressed in terms of the three-form flux in IIB, $G_{3}=F_{3}-\phi H_{3}$, as follows:

$$
G_{4}=-\frac{1}{\phi-\bar{\phi}} G_{3} \wedge d \bar{z}+\frac{1}{\phi-\bar{\phi}} \bar{G}_{3} \wedge d z .
$$

$G_{4}$ can also give rise to two-form flux, $F_{2}$, in the world-volume theory of the $D 7$ branes. For example, if two $D 7$-branes come together giving rise to an $A_{1}$ singularity and if $\alpha$ is the corresponding root of $\Gamma^{3,19}$ that is orthogonal to $\Omega$, then a non-trivial $F_{2}$ is turned on in the relative $U(1)$ between the two $D 7$-branes if $G_{4}$ has a component of the form, $G_{4}=\beta \wedge \alpha$, where $\beta$ is an integral two-form in $K 3_{1}$. Note however that if $\operatorname{Pic}\left(K 3_{1}\right)$ is trivial - as will be the case generically - such a component is not allowed by supersymmetry. This follows from noting that since $\alpha$ is orthogonal to $\Omega$ it must be of type $(1,1)$. Supersymmetry requires that $G_{4}$ is of type $(2,2)$, this means $\beta$ must be an $(1,1)$ form in $K 3_{1}$ and must therefore belong to $\operatorname{Pic}\left(K 3_{1}\right)$. In any event, we shall avoid turning on such $F_{2}$ fluxes in our constructions.

3 The requirement that the Einstein metric on K3 is at an orbifold singularity is somewhat different. The metric corresponds to a choice of space-like three-plane and an orbifold singularity occurs if a root is orthogonal to this three-plane. This ensures that orbifold has not been resolved either by Kähler deformations or complex structure deformations. For F-theory we are only interested in the complex structure deformations which preserve the elliptic form and we therefore require orthogonality with respect to $\Omega$ and $U$. 


\section{Examples}

\subsection{Simplest examples}

We will now construct an explicit example where all the complex structure moduli are stabilised at a point of enhanced symmetry.

Consider the six-dimensional subspace $H_{3,3}=U \oplus U \oplus U$ of $H^{2}(K 3, Z)$. In a suitable basis, which we call $\left(e_{1}, \cdots e_{6}\right)$ the inner product in this subspace takes the form, $2 \eta_{3,3}$, with $\eta_{3,3}=\operatorname{diag}(1,1,1,-1,-1,-1)$. The basis elements are chosen so that $e_{1}, e_{4}$ span the first $U$ sublattice and so on. Also we note that $e_{1}, e_{2}, e_{3}$ are space-like and the rest are time-like.

Now consider the flux

$$
\frac{G_{4}}{2 \pi}=e_{1} \wedge \tilde{e}_{1}+e_{2} \wedge \tilde{e}_{2}
$$

where $e_{1}, e_{2}$ and $\tilde{e}_{1}, \tilde{e}_{2}$ refer to vectors in the integral lattice of $K 3_{1}$ and $K 3_{2}$ respectively. This flux satisfies the requirement of having two legs along the two K3s respectively.

It is easy to see that $G_{4}$ can be written as

$$
\frac{G_{4}}{2 \pi}=\frac{1}{2}\left[\left(e_{1}+i e_{2}\right) \wedge\left(\tilde{e}_{1}-i \tilde{e}_{2}\right)+\left(e_{1}-i e_{2}\right) \wedge\left(\tilde{e}_{1}+i \tilde{e}_{2}\right)\right]
$$

So by choosing the complex structure $\Omega_{1}, \Omega_{2}$ of $K 3_{1}, K 3_{2}$ as follows,

$$
\begin{gathered}
\Omega_{1}=\left(e_{1}+i e_{2}\right) \\
\Omega_{2}=\left(\tilde{e}_{1}+i \tilde{e}_{2}\right),
\end{gathered}
$$

we see that

$$
\frac{G_{4}}{2 \pi}=\frac{1}{2}\left[\Omega_{1} \wedge \bar{\Omega}_{2}+\bar{\Omega}_{1} \wedge \Omega_{2}\right]
$$

and is therefore of type $(2,2)$. We note that the identification $(4.3),(4.4)$ is consistent with requiring that $\Omega_{1,2}$ are space-like two-planes in $H^{2}\left(K 3_{1,2}, R\right)$. Also, since, $e_{1} \cdot e_{1}=$ $e_{2} \cdot e_{2}, e_{1} \cdot e_{2}=0$ and similarly for $\tilde{e}_{1,2},(4.3)$, (4.4) are consistent with the requirements that $\Omega_{1} \cdot \Omega_{1}=\Omega_{2} \cdot \Omega_{2}=0$. In addition note that $\tilde{e}_{3}, \tilde{e}_{6}$ span a subspace $U$ of $H^{2}(K 3, Z)$ and are orthogonal to $\Omega_{2}$. This ensures that $K 3_{2}$ is elliptically fibered.

$G_{4}$ is orthogonal to $\tilde{e}_{3}, \tilde{e}_{6}$ and therefore to $U$, this ensures that one leg of $G_{4}$ is along the base and the other along the fiber of $K 3_{2}$.

Finally a Kähler form for $K 3_{1}$ can be chosen meeting the condition (2.1). The Kähler form corresponds to a space-like direction in $H^{2}(K 3, R)$ orthogonal to $\Omega_{1}$. In the example 
above we could take this direction to be along $e_{3}$, then we see that $e_{3} \cdot G_{4}=0$ so that the condition of primitivity is met.

Thus we see that all the requirements for an $\mathcal{N}=1$ solution are met in this example.

We will argue next that the complex structure moduli are all frozen about this point. Consider a small deformation in the complex structure of $K 3_{1}$. Under it $\Omega_{1} \rightarrow \Omega_{1}+\chi_{1}$ where $\chi_{1}$ is a $(1,1)$ form in $K 3_{1}$. Similarly $\Omega_{2} \rightarrow \Omega_{2}+\chi_{2}$. Under this transformation,

$$
\frac{G_{4}}{2 \pi}=\frac{1}{2}\left[\Omega_{1} \wedge \bar{\Omega}_{2}+\bar{\Omega}_{1} \wedge \Omega_{2}+\chi_{1} \wedge \bar{\chi}_{2}+\bar{\chi}_{1} \wedge \chi_{2}+K_{4}\right]
$$

with the four form $K_{4}$ defined as

$$
K_{4}=\chi_{1} \wedge \bar{\Omega}_{2}+\bar{\chi}_{1} \wedge \Omega_{2}+\chi_{2} \wedge \bar{\Omega}_{1}+\bar{\chi}_{2} \wedge \Omega_{1}
$$

Now since, $\Omega_{1,2}, \bar{\Omega}_{1,2}$ are linearly independent, $K_{4}$ cannot vanish, so $G_{4}$ cannot be of type $(2,2)$ after the small deformation. Thus for small deformations, $G_{4}$ no longer remains $(2,2)$ and the complex structure moduli are all lifted.

Let us now turn to describing the enhanced symmetry. We note that the lattice vectors of $E_{8} \oplus E_{8} \subset \Gamma^{3,19}$ are orthogonal to $\Omega_{2}$. In addition they are orthogonal to the $U$ sublattice spanned by $\left(\tilde{e}_{3}, \tilde{e}_{6}\right)$. Similarly the vectors $\tilde{e}_{4}, \tilde{e}_{5}$ are roots which are orthogonal to both $\Omega_{2}$ and $U$. Thus the enhanced gauge symmetry in this example is $S U(2) \times S U(2) \times E_{8} \times E_{8}$.

Finally we note that the membrane tadpole condition is met in M-theory. Since

$$
\frac{1}{2} \int \frac{G}{2 \pi} \wedge \frac{G}{2 \pi}=4<\frac{\chi}{24}=24
$$

one will need to add 20 M2 branes (D3 branes) in M-theory (F-theory).

\subsection{An Orientifold example}

By starting with the example above and changing the flux one can alter the complex structure $\Omega_{2}$ so that the gauge symmetry is reduced. In particular the symmetry can be broken to $S O(8)^{4}$. The resulting model then corresponds to taking the elliptically fibered $K 3_{2}$ at the orientifold point, with 4 D7-branes at each orientifold plane. It is worth examining this orientifold limit of the example above in more detail. This will allow us to explicitly calculate the masses of the D7-branes. It will also allow us to make contact with the discussion in [24]. We will find below that the orientifold examples corresponds to solutions of the type $(2+, 0-)$ in the classification of [24] (section 3.3). 
It is quite straightforward to find flux that will stabilise the complex structure at an orientifold singularity. The complex structure moduli space of the elliptically fibered $K 3_{2}$ is 18 dimensional. At an orientifold point 16 of these moduli correspond to the location of D7-branes along the $T^{2}$ base of $K 3_{2}$ and the remaining two moduli are the dilaton-axion and the complex structure of the $T^{2}$. Requiring that the complex structure is at a $\left(D_{4}\right)^{4}$ singularity fixes the locations of the D7-branes while allowing the other two moduli to vary. We will proceed in two steps in the discussion below, first finding a particular point in the complex structure moduli space where the singularity is of $\left(D_{4}\right)^{4}$ type, and then determining a flux which fixes the complex structure at this point.

The model under consideration is dual to the heterotic string on $K 3 \times T^{2}$. It is well known that by turning on Wilson lines on the heterotic side one can break the gauge symmetry down to $S O(8)^{4}$. Using the duality map, one can then map this to a location in the complex structure moduli space of $K 3_{2}$. Before proceeding let us note that, upto a sign convention, our discussion of Wilson lines in the heterotic string will be based on [31].

For simplicity we take the heterotic theory with a square $T^{2}$ at the self-dual point, with no $B$ field and with appropriate Wilson lines turned on. The resulting complex structure of $K 3_{2}$ can then be described as follows. The complex structure of $K 3_{2}$ corresponds to a twoplane $\Omega_{2}$. In $\S 4.1$ we described the basis vectors $e_{1}, \cdots e_{6}$ of the subspace $H_{3,3} \subset \Gamma^{3,19}$, with $e_{1}, e_{4}$ spanning the first $U$ subspace etc. It is easy to see that $n_{1}=\frac{e_{1}+e_{4}}{2}$ is a null vector, $n_{1} \cdot n_{1}=0$, meeting the condition $n_{1} \cdot e_{1}=1$. Furthermore it is a basis element for the $U$ sublattice of $\Gamma^{3,19}$. Similarly, we define the null vector, $n_{2}=\frac{e_{2}+e_{5}}{2}$, which is a lattice basis element for the second $U$ sublattice of $H_{3,3}$. We will also need to introduce a basis in the $\left(-E_{8}\right) \oplus\left(-E_{8}\right)$ sublattice of $\Gamma^{3,19}$. This is done by choosing vectors, $E_{I}, I=1 \cdots 16, E_{I} \cdot E_{J}=-\delta_{I J}$. The roots of $\left(-E_{8}\right) \oplus\left(-E_{8}\right)$ are then given by $q^{I} E_{I}$ for suitably chosen $q^{I}$ as discussed in Appendix B. The required spacelike two plane corresponds to a choice of two linearly independent space-like vectors. These are given by $\hat{\tilde{e}}_{1}, \hat{\tilde{e}}_{2}$ respectively, where

$$
\begin{aligned}
& \hat{\tilde{e}}_{1}=\tilde{e}_{1}+W_{I} E_{I}+\frac{\tilde{n}_{1}}{2} W_{I} W_{I} \\
& \hat{\tilde{e}}_{2}=\tilde{e}_{2}+\tilde{W}_{I} E_{I}+\frac{\tilde{n}_{2}}{2} \tilde{W}_{I} \tilde{W}_{I}
\end{aligned}
$$

Here $W_{I}, \tilde{W}_{I}$ denote the two Wilson lines. Also as in the previous section, we are following conventions where the tilde superscript as in $\tilde{e}_{1}$ etc, refers to elements of $H^{2}\left(K 3_{2}\right), \Gamma^{3,19}\left(K 3_{2}\right)$. With

$$
W_{I}=\operatorname{diag}\left(1,0^{7}, 1,0^{7}\right)
$$




$$
\tilde{W}_{I}=\operatorname{diag}\left(0^{4}, \frac{1}{2}^{4}, 0^{4}, \frac{1}{2}^{4}\right),
$$

one can show that the roots of $\Gamma^{3,19}$ orthogonal to $\hat{\tilde{e}}_{1}, \hat{\tilde{e}}_{2}$ correspond to the gauge group $S O(8)^{4}$. It is easy to see that $\hat{\tilde{e}}_{1}, \hat{\tilde{e}}_{2}$ are linearly independent and therefore define a spacelike two-plane. Identifying this two-plane with $\Omega_{2}$ gives the location of a $\left(D_{4}\right)^{4}$ singularity in the complex structure moduli space of $K 3_{2}$.

We now turn to determining the required flux which will stabilise the complex structure at this point in moduli space. As discussed in appendix B, $2 \hat{\tilde{e}}_{1}, 2 \hat{\tilde{e}}_{2}$, are elements of the integral lattice, $\Gamma^{3,19}$. So we can consider turning on the four-form flux,

$$
G_{4}=2 e_{1} \wedge \hat{\tilde{e}}_{1}+2 e_{2} \wedge \hat{\tilde{e}}_{2}
$$

Since $\hat{\tilde{e}}_{1}, \hat{\tilde{e}}_{2}$ satisfy the relations $\hat{\tilde{e}}_{1} \cdot \hat{\tilde{e}}_{1}=2, \hat{\tilde{e}}_{2} \cdot \hat{\tilde{e}}_{2}=2, \hat{\tilde{e}}_{1} \cdot \hat{\tilde{e}}_{2}=0$, we see that the discussion in the previous section goes through unchanged showing that a complex structure for $K 3_{1} \times K 3_{2}$ exists given by (4.5), with $G_{4}$ being of type $(2,2) . \Omega_{1}$ is unchanged from (4.3), and $\Omega_{2}$ is given by

$$
\Omega_{2}=\hat{\tilde{e}}_{1}+i \hat{\tilde{e}}_{2}
$$

Let us end this section with a few comments. First, since $G_{4}$ is of form (4.5), our discussion in the previous section still goes through leading to the conclusion that the complex structure moduli are all stabilised. Second, we note that $G_{4}$ above does not have any component along the root lattice of $S O(8)^{4}$, thus no gauge field flux $F_{2}$ is turned on in this case along the 7-brane world volumes. Third, the total contribution to the membrane tadpole condition is 16 , this means $8 D 3$ branes would have to be added in the F-theory description. Fourth, the fact that this example maps to the $(2+, 0-)$ case in [24] as mentioned above, follows simply by noting that there are only two linearly independent two-forms, $e_{1}, e_{2}$ of $K 3_{1}$ in $G_{4}$. Finally, for simplicity here we have focused on one choice of flux. More generally other choices of flux can also stabilise the complex structure at a $\left(D_{4}\right)^{4}$ singularity for other values of the dilaton-axion and $\tau$.

\section{The orientifold model in more detail}

It is worth exploring the orientifold model above in some more detail. We have given a general argument above that all the complex structure moduli are stabilised. Here we would like to explicitly verify this for the D7 moduli by calculating their mass. This will allow us to calculate in the next section the leading contribution (at large volume) to the non-perturbative superpotential due to gaugino condensation. 


\subsection{Dr-moduli mass}

The complex structure moduli space of the elliptically fibered $K 3_{2}$ is 18 complex dimensional. At the orientifold point, 16 of these 18 moduli correspond to Wilson lines that give the location of the $D 7$-branes along the base $T^{2}$ of $K 3_{2}$. The remaining two moduli correspond to the dilaton-axion, and the modular parameter of the base $T^{2}$. At the orientifold point, as was mentioned above, $4 \mathrm{D} 7$ branes are located at each O7-plane. By symmetry one can see that all the D7-branes must have the same mass. Displacing one $D 7$-brane from the O7-plane breaks the symmetry to $S O(6) \times S O(8)^{3}$. The corresponding Wilson lines are given by

$$
\begin{gathered}
W_{I}=\operatorname{diag}\left(1, \alpha, 0^{6}, 1,0^{7}\right), \\
\tilde{W}_{I}=\operatorname{diag}\left(0, \beta, 0^{2}, \frac{1^{4}}{2}, 0^{4}, \frac{1^{4}}{2}\right),
\end{gathered}
$$

where $\alpha, \beta$ are the locations of the $D 7$-brane along the $T^{2}$. The resulting complex structure of $K 3_{2}$ is given by (4.11), where $\hat{\tilde{e}}_{1}, \hat{\tilde{e}}_{2}$ are now given by

$$
\begin{aligned}
& \hat{\tilde{e}}_{1}=\tilde{e}_{1}+W_{I} E_{I}+\frac{\tilde{n}_{1}}{2} W_{I} W_{I}+\frac{\tilde{n}_{2}}{2} W_{I} \tilde{W}_{I}, \\
& \hat{\tilde{e}}_{2}=\tilde{e}_{2}+\tilde{W}_{I} E_{I}+\frac{\tilde{n}_{2}}{2} \tilde{W}_{I} \tilde{W}_{I}+\frac{\tilde{n}_{1}}{2} W_{I} \tilde{W}_{I},
\end{aligned}
$$

with the Wilson lines (5.1), (5.2). As a check it is easy to see that this complex structure corresponds to an unbroken $S O(6) \times S O(8)^{3}$ symmetry.

We now turn to determining the mass for the D7-brane moduli. The superpotential is given by

$$
W=\int G_{4} \wedge \Omega_{4}
$$

$G_{4}$ is fixed and given by (4.10), with $\hat{\tilde{e}}_{1}, \hat{\tilde{e}}_{2}$ being given by (4.6), (4.7), with the Wilson lines, (4.8), (4.9), respectively. $\Omega_{4}=\Omega_{1} \wedge \Omega_{2}$. As the 7 -brane moves away from the O7 plane the complex structure of $K 3_{2}, \Omega_{2}$, changes, as described in the previous paragraph. $\Omega_{1}$ is fixed and given by (4.3). By substituting these expressions in $W$ above it is straightforward to see that it takes the form,

$$
W=4(\alpha+i \beta)^{2} .
$$

We saw above that the complex scalar $\alpha+i \beta$ is the location of the D7-brane along the $T^{2}$ base of $K 3_{2}$. We will denote it by $\Phi=\alpha+i \beta$ in the discussion below. The quadratic term in the superpotential shows that this modulus acquires a mass. From (5.5), it is clear that the mass is linear in the flux.

One final comment. Our result above for the 7-brane moduli mass agrees with an earlier calculation using the methods of gauged supergravity, [26]. 


\subsection{The Non-perturbative Superpotential}

The low-energy dynamics of the orientifold model discussed above is that of an $\mathcal{N}=1$ $S O(8)^{4}$ gauge theory. The gauge group arises form the gauge fields on the D7-branes. The D7-branes wrap $K 3_{1}$ and are transverse to the $T^{2}$ base of $K 3_{2}$. The D7-brane locations on the $T^{2}$ are moduli which are adjoint chiral superfields in the gauge theory. There is one adjoint field for each $S O(8)$ group. We saw above that these moduli acquire a mass due to the flux. This mass scales with the radius of compactification, $R$, as $1 / R^{3}$. At energy scales below this mass scale the low-energy dynamics is that of a pure $\mathcal{N}=1 \mathrm{SYM}$ theory with gauge group $S O(8)^{4}$. It is well known that a non-perturbative superpotential is generated in pure SYM theory due to gaugino condensation. The form of this superpotential can be determined by standard field theory techniques. Since the gauge fields arise from the D7-brane world volume, the gauge coupling is given by

$$
S=\frac{8 \pi^{2}}{g_{Y M}^{2}}+i \theta=e^{4 u-\phi}+i b,
$$

where $e^{4 u}$, is the volume of $K 3_{1}, \phi$ is the dilaton and $b$ is an axion that arises from the $\mathrm{RR}$ four-form. The gauge couplings of the four $S O(8)$ groups are the same.

Standard field theory techniques then show that the non-perturbative superpotential is given by :

$$
W_{N P}=A e^{-S / c_{2}} m
$$

where $m$ is the mass of the adjoint chiral superfield. $A$ above is a coefficient that depends on the expectation values of the frozen complex structure moduli, and $c_{2}$ is the dual Coxeter number in the adjoint representation of the gauge group. For $S O(2 n), c_{2}=(2 n-2)$, giving in particular $c_{2}=6$ for $S O(8)$.

Let us briefly sketch how this result is obtained. We denote the tree-level superpotential, (5.6), as $W_{\text {tree }}$ in the discussion below. This tree-level superpotential has a $U(1)$ R-symmetry under which $\Phi \rightarrow \Phi e^{i \theta}$. The R-symmetry corresponds to rotations in the plane of the $T^{2}$ in $K 3 \times T^{2} / Z_{2}$. We see that $W_{\text {tree }}$ has charge 2 under this R-symmetry. This symmetry is anomalous in the quantum theory and it is easy to see that $S \rightarrow S-2 i c_{2} \theta$ under it. Now $W_{N P}$ must transform in the same way as $W_{\text {tree }}$ under this symmetry, this fixes the dependence on $S$ in (5.7). Similarly $W_{\text {tree }}$ has an R-symmetry under which 
$m$ transforms with charge 2 and $\Phi$ is invariant. The R-symmetry can be shown to be non-anomalous so $S$ is invariant under it. This determines the $m$ dependence in $(5.7)^{4}$.

The non-perturbative superpotential (5.7) results in a potential for the volume modulus of $K 3_{1}$. In general one would expect that (5.7) is not exact in string theory and there are corrections to it which are subleading at large volume. Requiring that $W_{N P}$ transforms correctly under full duality group, together with the large volume dependence determined above, might help fix the form of these subleading corrections.

\section{Divisors contributing to the superpotential}

Let us begin by reminding the reader of Witten's argument [10] that divisors $D$ of arithmetic genus, $\chi=1$, are the relevant ones for superpotential generation in M-theory compactification on a Calabi-Yau fourfold $X$. For a Euclidean brane instanton to contribute to the superpotential, it should break half of the space-time supersymmetry, leaving precisely two fermion zero modes. For a Euclidean M5 brane instanton the zero modes are determined by the $h^{k, 0}(D)$ cohomology groups of the divisor wrapped by the Euclidean 5-brane. Witten considered the $U(1)$ symmetry of the normal bundle $N$ to $D$ and argued that modes arising from the cohomology groups $h^{2 p, 0}(D)$ and $h^{2 p+1,0}(D)$ have opposite $U(1)$ charges and can pair up. The index obtained after grading the zero modes by the sign of their charge under this $U(1)$ symmetry is $2 \chi$. Thus a necessary (although not always sufficient) condition for the non-perturbative superpotential to arise is that $\chi=1$. We will see that after accounting for the effects of G-flux, this conclusion is modified, and in general $\chi>1$ divisors can also contribute to the space-time superpotential; the loophole was anticipated on p.10 of [10].

\subsection{In the $K 3 \times K 3$ examples}

Here, we describe in slightly more detail the geometry of the singular $K 3 \times K 3$ compactifications that must be yielding our gaugino condensates. In each of these cases, the singular elliptic $K 3_{2}$ has an A-D-E singularity. For simplicity we will focus on the case of

4 These two R symmetries act on the GVW superpotential as follows. Under the first symmetry, $\Omega \rightarrow \Omega e^{2 i \theta}$, and the flux $G_{4}$ is invariant. Under the second symmetry, $G_{4} \rightarrow G_{4} e^{2 i \theta}$, and $\Omega$ is invariant. Towards the end of section 6 we will refer to a $U(1)$ discussed in [10]. This symmetry is a combination of the two R-symmetries discussed above. Under it, with our choice of normalisation, $\Phi$ and $m$ have charges $2,-2$, respectively, while $\Omega, G_{4}$, have charges $4,-2$. 
$A_{N-1}$ here, but very similar remarks apply to the other two cases. Our discussion of the relevant geometries follows [23].

We have an $A_{N-1}$ singularity over $K 3_{1}$. Denote the exceptional divisor by $D . D$ is the union of $N-1$ irreducible components $D=\cup_{i} D_{i}$. After blowing up, the fiber over $K 3_{1}$ will consist of $N P^{1} \mathrm{~s}$, intersecting in such a way as to form the affine Dynkin diagram for $S U(N)$ (the $D_{i}$ have been supplemented by an additional divisor $D^{\prime}$, which is the closure of the complement of the exceptional set $D$ inside the resolved elliptic fiber ${ }^{5}$.

In general examples that would arise in elliptic Calabi-Yau fourfolds, this story would generalize as follows. One would look for singularities of the elliptic fibration of Kodaira $I_{N}$ type over some surface $S$ in the base $B$ (the singularities may worsen to $I_{N+1}$ at codimension one in $S$, etc.). The $D_{i}$ (and $D^{\prime}$ ) are then nontrivial $P^{1}$ bundles over the surface $S$ wrapped by the D7 branes $\pi: D_{i} \rightarrow S$. Our particular example is however quite simple: $S$ is $K 3_{1}$ and the fiber is constant along $S$. Therefore, the $D_{i}$ simply take the form $K 3_{1} \times P^{1}$. It is easy to see that the arithmetic genus of these cycles satisfies

$$
\chi\left(D_{i}, \mathcal{O}\left(D_{i}\right)\right)=h^{0,0}(K 3)+h^{2,0}(K 3)=2 .
$$

Hence, while it is completely clear from the $4 \mathrm{~d}$ and $10 \mathrm{~d}$ perspectives that our examples have pure Yang-Mills sectors which will undergo gaugino condensation, there need not be divisors of arithmetic genus 1 . This is in keeping with the remarks in [10] about how infrared gauge theory effects may not give the correct naive zero mode count required for superpotential generation.

It is suggestive that an M5-brane wrapping $D$ (i.e., all $N P^{1} \mathrm{~s}$ ) would be wrapping a cycle of $\chi(D)=2 N$. This coincides with the number of fermion zero modes which are present in a naive instanton calculation in pure $S U(N) \mathcal{N}=1$ Yang-Mills theory, where the gaugino condensate $\langle\lambda \lambda\rangle$ scales like the $N$ th root of a gaugino $2 \mathrm{~N}$-point correlator (see e.g. [32], pages 184-186). This underscores once again the fact that gaugino condensation is not an instanton effect in the $4 \mathrm{~d}$ field theory picture, and it is not very surprising that there is no instanton with two fermion zero modes.

5 The additional $P^{1}$ which gives rise to the divisor $D^{\prime}$ can be understood as follows. Since $K 3_{2}$ is elliptically fibered there is a null vector, $n$, dual to the $T^{2}$ fiber. Like the roots of $A_{N-1}$, $e_{i}, n$ is also orthogonal to the the two-plane, $\Omega_{2}$. The additional $P^{1}$ is dual to $n-\sum e_{i}$. And $e_{i}$ together with $n-\sum_{i} e_{i}$ give rise to the Dynkin diagram of affine $A_{N-1}$. 


\subsection{More general cases}

More generally, our construction suggests the following. Consider any case where in a fourfold, there is a Kodaira type $I_{N}$ degeneration over $S$ as described above (in physics language, this is the situation when there are $N$ D7 branes wrapping $S$ ). In general there will be $I_{N+1}$ curves in $S$ also (physically, these are curves where another D7 intersects the stack of $N$ D7s wrapping $S$ ). One can then consider an M5 brane wrapping the cycles $D_{i} \rightarrow S$ and $D^{\prime} \rightarrow S$, which are fibrations over $S$ with $P^{1}$ fibers. Using the Leray spectral sequence as on p.6 of [23], we see the following. Since the $P^{1}$ fiber has $h^{1,0}\left(P^{1}\right)=0$, and the only holomorphic function on $P^{1}$ is a constant, $H^{i, 0}\left(D_{i}\right) \simeq H^{i, 0}(S)$. Therefore, these cycles will have arithmetic genus

$$
\chi\left(D_{i}\right)=h^{0,0}(S)-h^{1,0}(S)+h^{2,0}(S) .
$$

Let us assume for simplicity that $S$ is simply connected, so $h^{1,0}(S)=0$. This is not a serious restriction, as in many cases $S$ inherits its first cohomology from the cohomology of the base of the F-theory elliptic fourfold (by the Lefschetz hyperplane theorem), and for simple examples this vanishes. We then see that $\chi\left(D_{i}\right)>0$, and all zero modes but the one arising from $h^{0,0}(S)$ are in correspondence to adjoint matter fields on the D7 branes wrapping $S$. (Recall a D7 brane wrapping $S$ receives $h^{1,0}$ adjoint fields from Wilson lines on $S$, which vanishes for us, and $h^{2,0}$ adjoint fields from deformations of $S$ inside the compactification manifold). ${ }^{6}$ Hence in all cases where the matter fields are lifted by three-form fluxes leaving an $I_{N}$ degeneration over $S$, there are also cycles present of the appropriate arithmetic genus $(\chi \geq 1)$ to possibly contribute to the superpotential.

We therefore expect that in many examples of IIB compactifications with flux, one will obtain contributions to the nonperturbative superpotential from stabilized coincident D7 branes. This can happen sometimes even in the absence of cycles of arithmetic genus 1 in the related Calabi-Yau fourfold. However in all such cases, we expect (as in the $K 3 \times K 3$ examples) that divisors of $\chi \geq 1$ exist.

6 In general there can also be fundamental matter fields arising from the intersections with other D7s. These also yield a nonchiral spectrum in simple examples, and analogues of the phenomena we are exploring here should be expected to occur. Some examples of $S U\left(N_{c}\right)$ theories with quark flavors arising from D7s are described in [33]. In many cases, more exotic theories which are not yet well understood can also arise. 
We see no obstruction to such examples arising even in cases where the IIB compactification manifold has a single Kähler modulus. For instance, it is easy to write down examples in the elliptic fibration over $P^{3}$ which have singularities of various Kodaira types over a surface $S$ (which has $h^{1,0}(S)=0$ ) in $P^{3}$. The question is then whether appropriate fluxes can stabilize D7s on such a locus. If so, the resulting theory would exhibit gaugino condensation and a nonperturbative superpotential for the Kähler modulus. Our explicit examples of this in $K 3 \times K 3$ give us confidence that the phenomenon will happen in more general examples.

A recent paper [22] argued that vertical $\chi=1$ divisors cannot appear in elliptic fourfolds $X \rightarrow B$ with $h^{1,1}(B)=1$, and that hence such models cannot have nonperturbative superpotentials for the Kähler modulus. Since our comments suggest otherwise, let us address the contradiction. The arguments presented in [22] do not prove that vertical $\chi=1$ (or $\chi>1$ ) divisors cannot appear in models with $h^{1,1}(B)=1$. Equation (18) in $\S 2.5$ of [22], for the total Chern class of $X$, is in general incorrect for models where $h^{1,1}(X)>2$ but $h^{1,1}(B)=1$. This equation plays an important role in constraining the possible arithmetic genera of divisors. The argument of $\S 2.6$ in [22], which shows that no base $B$ with $h^{1,1}(B)=1$ can be globally fibered over a surface $S$, is true. However, that fact is not relevant to the phenomena under discussion here. A global fibration of $B$ over some $S$ is not needed to obtain nonabelian gauge symmetries from coincident D7 branes in $B$, and it is straightforward to write down examples of $I_{n}$ singularities with $n>1$ in the fourfolds of [34] with $h^{1,1}(B)=1$.

In a forthcoming paper [35], the question of a non-perturbative superpotential in cases where the IIB compactification has a single Kähler modulus will be discussed in much greater depth. Several explicit examples of elliptic fourfolds $X \rightarrow B$ with $h^{1,1}(B)=1$ (and $h^{1,1}(X)>2$ ) and vertical divisors of arithmetic genus one have been found and will be presented in [35]. The simplest examples arise by working with the elliptic fourfold in $W P_{1,1,1,1,8,12}^{5}$ (which is elliptically fibered over $P^{3}$ ), constructing singularities of various Kodaira types over a $P^{2}$ in the $P^{3}$, and blowing them up.

Finally, let us close this section by noting that KKLT discussed the single Kähler modulus case solely for simplicity of exposition. The arguments presented there are more general and do not depend in any important way on this condition. 


\section{Discussion}

There are several obvious directions for further work. Needless to say, it would be interesting to find explicit examples of the phenomena we have seen in $K 3 \times K 3$ in other Calabi-Yau fourfolds. While explicit examples where all Kähler moduli can be stabilized already exist [11], we believe the kinds of effects described here will greatly broaden the class of examples.

It would also be interesting to understand the story when our models are reduced to $3 \mathrm{~d}$ directly in the language of M5 instantons. A $4 \mathrm{~d} \mathcal{N}=1$ pure gauge theory, reduced to $3 \mathrm{~d}$ on a circle, exhibits superpotential generation due to abelian instantons on the Coulomb branch, and a microscopic version of this involving M5 instantons appeared in [23]. Our situation involves a $4 \mathrm{~d}$ theory which has chiral multiplet matter fields, which are then given a mass. After the reduction to $3 \mathrm{~d}$, an instanton computation in the $3 \mathrm{~d}$ field theory must still yield a nonzero result: the mass terms for the matter fields can be pulled down to absorb any extra zero modes which naively appear in the instanton calculation. This macroscopic phenomenon must have a microscopic analogue in M5 instanton calculations, and it would be interesting to understand this in detail. One comment in this regard is worth making. As discussed in [10] the arithmetic genus of the divisor wrapped by the $M 5$ brane corresponds to an index graded by a $U(1)$ symmetry. This $U(1)$ symmetry is the structure group of the normal bundle of the divisor. In the $K 3_{1} \times K 3_{2}$ case, as is discussed further in appendix $\mathrm{C}$, one finds that the symmetry is broken by the flux. This suggests that in the M5 brane worldvolume theory, modes coming from $h^{2,0}$ can pair up amongst themselves and became massive in the presence of flux, allowing for a superpotential even when $\chi>1$.

Finally, we note that in our $K 3 \times K 3$ examples, $G_{4}$ fluxes which reduce purely to three-form fluxes in the IIB language (no field strengths $F_{2}$ turned on in the D7 branes) suffice to stabilize all D7 brane moduli. It would be interesting to see if this phenomenon persists in more generic examples. ${ }^{7}$

\section{Acknowledgements}

We would like to thank P. Aspinwall, F. Denef, O. DeWolfe, M. Douglas, N. Fakhruddin, B. Florea, A. Grassi, N. Iizuka, S. Katz, G. Moore, S. Shenker and A. Uranga for

7 It is clear that generic $G_{4}$ fluxes which reduce to both three-form fluxes and $F_{2}$ fluxes in IIB, do suffice to stabilize all D7 moduli in generic examples. This follows from simple counting of equations and fields given the GVW superpotential for a CY fourfold. 
helpful discussions. The work of S.K. was supported in part by a David and Lucile Packard Foundation Fellowship for Science and Engineering, the National Science Foundation, and the Department of Energy. S.P.T. acknowledges the support of the Swarnajayanti Fellowship, DST. Govt. of India. The work of L.G., P.K.T. and S.P.T. was supported by the Govt. of India, most of all they thank the people of India for enthusiastically supporting research in string theory.

\section{Appendix A. More on Elliptically Fibered $K 3$}

Here we will describe how the requirement that $U \subset P i c(K 3)$ arises for an elliptically fibered $K 3$.

In general, as we know, the second cohomology group for $K 3$ is given by the lattice

$$
\Gamma_{3,19}=U \oplus U \oplus U \oplus\left(-E_{8}\right) \oplus\left(-E_{8}\right)
$$

where $U$ is the hyperbolic plane, (3.1), and $E_{8}$ is the Cartan matrix for the $E_{8}$ lattice. Being an elliptic fibration, the $K 3$ obviously has, at least, two algebraic curves embedded in it, one being a nontrivial section of the bundle (which is guaranteed from the Weierstraß form) and the other being the elliptic fiber. Obviously, they are elements of the second homology group $H_{2}(K 3, Z)$ (which is same as $H^{2}(K 3, Z)$ ), and since they are algebraic they also belong to $H^{1,1}(K 3)$. Thus they belong to the Picard lattice of $K 3$.

We can obtain the intersection matrix for these two curves as follows. In general a genus $g$ curve in $K 3$ has self-intersection number equal to $(2 g-2)$ and hence for the section (which is a $P^{1}$ ) it is -2 , whereas for the fiber it is zero. Since both the curves intersect each other transversely, their intersection number is one. Thus the intersection matrix is

$$
\left(\begin{array}{cc}
-2 & 1 \\
1 & 0
\end{array}\right)
$$

which, after a change of basis, is identical to $U$. This means that the necessary condition for a $K 3$ of Weierstraß type is that the Picard lattice must contain $U$ as a sublattice. This also turns out to be a sufficient condition for the $K 3$ to admit an elliptic fibration.

It is clear from the discussion above that the two elements of $H^{2}(K 3, R)$ dual to the base and the fiber span $U$. Thus if $G_{4}$ must have one leg along the base and the other along the fibre of $K 3_{2}$ it cannot contain any element of the sublattice $U$. Since the matrix $U$ has eigenvalues $(1,-1)$, this means $G_{4}$ must be orthogonal to all vectors in $U$. 


\section{Appendix B. The $\left(-E_{8}\right) \oplus\left(-E_{8}\right)$ Lattice}

Here we discuss the $\left(-E_{8}\right) \oplus\left(-E_{8}\right)$ lattice in more detail. The discussion will be based on section 11.6 in Polchinski's book [31] with some changes in convention, to account for the time-like nature of the lattice in our case etc. As mentioned in the discussion above we take 16 vectors, $E_{I}, I=1, \cdots 16$ that are linearly independent and satisfy the the conditions $E_{I} \cdot E_{J}=-\delta_{I J}$. These vectors span the $\left(-E_{8}\right) \oplus\left(-E_{8}\right)$ vector space. The first $\left(-E_{8}\right)$ lattice is given by vectors of the form $\sum_{I=1}^{8} q^{I} E_{I}$, where the $\left(q^{1}, \cdots q^{8}\right)$ are either all integer or half integer and $\sum_{I} q^{I} \in 2 \mathbf{Z}$. The second $\left(-E_{8}\right)$ lattice is similarly given by appropriate linear combinations of $E_{I}, I=9, \cdots 16$.

It is now easy to see why $2 \hat{\tilde{e}}_{1}, 2 \hat{\tilde{e}}_{2},(4.6),(4.7)$, belong to the lattice $\Gamma^{3,19}$. $\tilde{e}_{1}, n_{1}$ belong to the first $U$ sublattice of $\Gamma^{3,19}$, as we discussed in section 3 and 4 , and similarly $\tilde{e}_{2}, n_{2}$ belong to the second $U$ sublattice. For the choice (4.8), (4.9), we have $W_{I} W_{I}=\tilde{W}_{I} \tilde{W}_{I}=2$. So to show that $2 \hat{\tilde{e}}_{1}, 2 \hat{\tilde{e}}_{2}$, belong to $\Gamma^{3,19}$ it is enough to show that $2 W_{I} E_{I}$ and $2 \tilde{W}_{I} E_{I}$ belong to it. But we see that both of these vectors are of the form $q^{I} E_{I}$ with $q^{I}$ meeting the conditions mentioned above, so this is true.

\section{Appendix C. Flux Breaks the M5 Brane Worldvolume $U(1)$ Symmetry}

As discussed in $\S 6$ the arithmetic genus of the divisor wrapped by the Euclidean M5 brane corresponds to an index graded by the $U(1)$ symmetry which is the structure group of the normal bundle of the divisor. Here we show that in the $K 3_{1} \times K 3_{2}$ case this $U(1)$ symmetry is broken by the $G_{4}$ flux.

Roughly speaking the argument is as follows. The divisor is $K 3_{1} \times P^{1}$, with the $P^{1}$ being an exceptional divisor of $K 3_{2}$ associated with blowing up an ADE singularity. As we saw above the flux, $G_{4}$, must have one leg along the fiber and one along the base of $K 3_{2}$. Now the singularity is located at a particular point in the base of $K 3_{2}$ so the tangent along the base is normal to the divisor. Thus $G_{4}$ breaks the $U(1)$ symmetry.

More precisely, let us illustrate this by taking the case of an $A_{1}$ singularity. In the vicinity of the singularity, $K 3_{2}$ is described by the equation,

$$
y^{2}=x^{2}-z^{2},
$$

in $C^{3}$, with the coordinate along the base of $K 3_{2}$ being say, $z$. Now the resolved $K 3_{2}$ is partially covered by the coordinates $z, s_{2}, s_{1}$, where $y=s_{2} z, x=s_{1} z$, and $s_{2}, s_{1}$, satisfy the relation

$$
s_{2}^{2}=s_{1}^{2}-1
$$


And the exceptional divisor in $K 3_{2}$ is partially covered by $z=0$ with $s_{2}, s_{1}$, satisfying $(\mathrm{C} .1)^{8}$.

Away from $z=0$, the tangent vector along the the base $P^{1}$ is given by $\partial_{z}$, and the tangent vector along the fiber $T^{2}$ by $\partial_{s_{1}}+\frac{\partial s_{2}}{\partial s_{1}} \partial_{s_{1}}$. So it is clear that as $z \rightarrow 0$, and we approach the divisor, the tangent vector along the fiber becames the tangent vector to the divisor and the tangent vector along the base becames the normal to the divisor. Since, as we mentioned above, $G_{4}$ has one leg along the base and one along the fiber of $K 3_{2}$, we see that the flux breaks the $U(1)$ symmetry.

The argument above clearly generalises to other ADE singularities in $K 3_{2}$ as well.

8 These coordinates miss two points in the resolved $K 3_{2}$. The divisor is actually the surface $s^{2}=s_{1}^{2}-s_{3}^{2}$ in $P^{2}$, and the two points not included are $s_{3}=0, s_{2}= \pm s_{1}$. 


\section{References}

[1] R. Bousso and J. Polchinski, "Quantization of Four-Form Fluxes and Dynamical Neutralization of the Cosmological Constant," JHEP 0006 (2000) 006, hep-th/0004134.

[2] A. Maloney, E. Silverstein and A. Strominger, "De Sitter Space in Noncritical String Theory," hep-th/0205316.

[3] S. Kachru, R. Kallosh, A. Linde and S. P. Trivedi, "De Sitter Vacua in String Theory," Phys. Rev. D68 (2003) 046005, hep-th/0301240.

[4] L. Susskind, "The Anthropic Landscape of String Theory," hep-th/0302219.

[5] M. Douglas, "The Statistics of String/M theory vacua," JHEP 0305 (2003) 046, hepth/0303194.

[6] E. Silverstein, "TASI/PITP/ISS Lectures on Moduli and Microphysics," hep-th/0405068;

V. Balasubramanian, "Accelerating Universes and String Theory," hep-th/0404075;

A. Frey, "Warped Strings: Self-Dual Flux and Contemporary Compactifications," hepth/0308156.

[7] S. Giddings, S. Kachru and J. Polchinski, "Hierarchies From Fluxes in String Compactifications," Phys. Rev. D66 (2002) 106006, hep-th/0105097.

[8] K. Becker and M. Becker, "M-theory on Eight Manifolds," Nucl. Phys. B477 (1996) 155, hep-th/9605053;

S. Sethi, C. Vafa and E. Witten, "Constraints on low-dimensional string compactifications," Nucl. Phys. B480 (1996) 213, hep-th/9606122.

[9] S. Gukov, C. Vafa and E. Witten, "CFTs from Calabi-Yau Fourfolds," Nucl. Phys. B584 (2000) 69, hep-th/9906070; T. Taylor and C. Vafa, "RR Flux on Calabi-Yau and Partial Supersymmetry Breaking," Phys. Lett. B474 (2000) 130, hep-th/9912152; P. Mayr, "On Supersymmetry Breaking in String Theory and its Realization in Brane Worlds," Nucl. Phys. B593 (2001) 99, hep-th/0003198.

[10] E. Witten, "Nonperturbative superpotentials in string theory," Nucl. Phys. B474 (1996) 343, hep-th/9604030.

[11] F. Denef, M. Douglas and B. Florea, "Building a Better Racetrack," JHEP 0406 (2004) 034, hep-th/0404257.

[12] I. Klebanov and M. Strassler, "Supergravity and a confining gauge theory: Duality cascades and $\chi$ SB resolution of naked singularities," JHEP 0008 (2000) 052, hepth/0007191.

[13] S. Kachru, J. Pearson and H. Verlinde, "Brane/Flux Annihilation and the String Dual of a Non-Supersymmetric Field Theory," JHEP 0206 (2002) 021, hep-th/0112197.

[14] C.P. Burgess, R. Kallosh and F. Quevedo, "de Sitter string vacua from supersymmetric D-terms," JHEP 0310 (2003) 056, hep-th/0309187.

[15] A. Saltman and E. Silverstein, "The scaling of the no-scale potential and de Sitter model building," hep-th/0402135. 
[16] R. Brustein and S. de Alwis, "Moduli potentials in string compactifications with fluxes: Mapping the discretuum," hep-th/0402088.

[17] S. Ashok and M. Douglas, "Counting Flux Vacua," JHEP 0401 (2004) 060, hepth/0307049.

[18] F. Denef and M. Douglas, "Distributions of Flux Vacua," JHEP 0405 (2004) 072, hep-th/0404116.

[19] A. Giryavets, S. Kachru and P. Tripathy, "On the Taxonomy of Flux Vacua," hepth/0404243.

[20] S. Kachru, unpublished.

[21] A. Grassi, "Divisors on elliptic Calabi-Yau fourfolds and the superpotential in F-theory I," J. Geom. Phys. 28 (1998) 289.

[22] D. Robbins and S. Sethi, "A Barren Landscape," hep-th/0405011.

[23] S. Katz and C. Vafa, "Geometric Engineering of $\mathcal{N}=1$ Quantum Field Theories," Nucl. Phys. B497 (1997) 196, hep-th/9611090.

[24] P. K. Tripathy and S. P. Trivedi, "Compactifications with Flux on K3 and Tori," JHEP 0303 (2003) 028, hep-th/ 0301139.

[25] G. Moore, "Les Houches Lectures on Strings and Arithmetic," hep-th/0401049.

[26] C. Angelantonj, R. D'Auria, S. Ferrara and M. Trigiante, "K3 x T**2/Z(2) orientifolds with fluxes, open string moduli and critical points," Phys. Lett. B583 (2004) 331, hepth/0312019.

[27] J.F. Cascales and A. Uranga, "Branes on Generalized Calibrated Submanifolds," to appear.

[28] P. Camara, L. Ibanez and A. Uranga, "Flux-induced SUSY-breaking soft terms on D3-D7 systems," to appear;

P. Camara, L. Ibanez and A. Uranga, "Flux-induced SUSY-breaking soft terms," Nucl. Phys. B689 (2004) 195, hep-th/0311241.

[29] P. S. Aspinwall, "K3 Surfaces and String Duality," hep-th/9611137.

[30] K. Dasgupta, G. Rajesh and S. Sethi, "M Theory, Orientifolds and G-Flux," JHEP 9908 (1999) 023, hep-th/9908088.

[31] J. Polchinski, String Theory, Volume II, Cambridge University Press, 1998.

[32] D. Amati, K. Konishi, Y. Meurice and G. Veneziano, "Nonperturbative Aspects in Supersymmetric Gauge Theories," Phys. Rept. 162 (1988) 169.

[33] D. Diaconescu and S. Gukov, "Three Dimensional $N=2$ Gauge Theories and Degenerations of Calabi-Yau Fourfolds," Nucl. Phys. B535 (1998) 171, hep-th/9804059.

[34] A. Klemm, B. Lian, S.S. Roan and S.T. Yau, "Calabi-Yau Fourfolds for M-theory and F-theory Compactifications," Nucl. Phys. B518 (1998) 515, hep-th/9701023.

[35] F. Denef, M. Douglas, B. Florea and S. Kachru, to appear. 